\title{
ExoU-induced procoagulant activity in Pseudomonas aeruginosa-infected
} airway cells

\author{
M.C. Plotkowski, L.F.P. Feliciano, G.B.S. Machado, L.G. Cunha Jr, C. Freitas,
} A.M. Saliba and M.C. de Assis

ABSTRACT: The present study addressed the question whether ExoU, a Pseudomonas aeruginosa toxin with phospholipase A2 (PLA2) activity, may induce airway epithelial cells to overexpress tissue factor (TF) and exhibit a procoagulant phenotype.

Cells from the human bronchial epithelial BEAS-2B line were infected with an ExoU-producing $P$. aeruginosa strain, pre-treated or not with the cytosolic PLA2 inhibitor methylarachidonyl fluorophosphate (MAFP), or with two ExoU-deficient mutants. Control noninfected and infected cells were assessed for the expression of: 1) TF mRNA by RT-PCR; 2) cell-associated TF by enzyme immunoassay and flow cytometry; 3) procoagulant activity by a colorimetric assay; and 4) microparticle-associated TF by flow cytometry. An enzyme immunoassay was also used to assess cell-associated TF in lung extracts from mice infected intratracheally with ExoU-producing and deficient bacteria.

Cells infected with the wild-type bacteria had higher levels of TF mRNA, cell-associated TF expression, procoagulant activity and released microparticle-associated TF than cells infected with the mutants. Bacterial treatment with MAFP significantly reduced the expression of TF by infected cells. Lung samples from mice infected with the wild-type bacteria exhibited higher levels of cell-associated TF and procoagulant activity.

The present results demonstrate that ExoU may contribute to the pathogenesis of lung injury by inducing a tissue factor-dependent procoagulant activity in airway epithelial cells.

KEYWORDS: ExoU, lung injury, procoagulant activity, Pseudomonas aeruginosa, sepsis, tissue factor

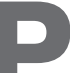
seudomonas aeruginosa is one of the leading causes of Gram-negative nosocomial pneumonia. P. aeruginosa hospitalacquired pneumonia often results in sepsis, a severe clinical syndrome characterised by a generalised activation of inflammation and coagulation pathways.

The lungs are among the most frequently affected organs during sepsis [1], and characteristically show fibrin deposition in alveolar and interstitial compartments [2]. Besides compromising the lung gas-exchange barrier, alveolar clotting processes are harmful because neutrophils and fibroblasts may be activated further by thrombin and fibrin degradation products, contributing to further tissue damage. Moreover, surfactant components may be incorporated into polymerising fibrin with subsequent loss of surface activity and alveolar instability [3]. However, the mechanisms that control fibrin deposition in patient airways remain poorly understood.
Tissue factor (TF; CD142), the major physiological initiator of the coagulation cascade, is an integral membrane protein expressed on a number of cells not in direct contact with blood. However, in both monocytes and endothelial cells, TF can be readly up-regulated by proinflammatory cytokines, bacterial lipopolysaccharide (LPS) and many other proinflammatory stimuli [4], giving these cells a prothrombotic phenotype. TF is fully functional when expressed on cell surfaces and binds factor VII, supporting its allosterical activation to factor VIIa. The enzymatic TF-VIIa complex then initiates the downstream clotting events that culminate in the conversion of prothrombin to thrombin. Thrombin proteolytically cleaves fibrinogen, yielding fibrin monomers that polymerise into a stable clot required for physiological haemostasis.

TF is also present in human tissues with large procoagulant activity, such as brain and placenta, as well as in multiple lung cells, including

\section{AFFILIATIONS}

Departamento de Microbiologia Imunologia e Parasitologia, Faculdade de Ciências Médicas, Universidade do Estado do Rio de Janeiro, Rio de Janeiro, Brazil.

CORRESPONDENCE

M.C. Plotkowski

Departamento de Microbiologia

Imunologia e Parasitologia

Faculdade de Ciências Médicas

UERJ

Av. 28 de Setembro

87 fundos

$3^{\circ}$ andar. $20551-030$

Rio de Janeiro

Brazil

Fax: 552125876476

E-mail: crisplot@yahoo.com.br

Received

June 072008

Accepted after revision:

July 192008

\section{SUPPORT STATEMENT}

The present study was supported by grants from Conselho Nacional de

Desenvolvimento Científico e

Tecnológico (Brasilia, Brazil; 470131/2006-3) and Fundação de Amparo à Pesquisa do Estado do Rio de Janeiro (Rio de Janeiro, Brazil; E26/100.587/2007 and $\mathrm{E}-26 /$ 1000.417/2007)

STATEMENT OF INTEREST

None declared. 
bronchoalveolar macrophages and alveolar and bronchial epithelial cells $[5,6]$. TF expression in alveolar cells has long been known to be modulated by inflammatory stimuli [4, 7], but the ability of the alveolar epithelium to initiate intraalveolar coagulation and fibrin deposition through upregulation of active TF has only been addressed recently [8].

TF can also be released from different cell types, and circulates in extracellular fluids as a soluble fluid-phase protein [9] or associated with microparticles [10, 11]. Microparticles are vesicles shed from the blebbing plasma membrane of various cell types. In body fluids, they constitute reliable hallmarks of cell activation and/or damage. Microparticles exhibit anionic phospholipids, chiefly phosphatidylserine, at their surface, which provide a catalytic surface promoting the assembly of the enzyme complexes of the coagulation cascade. Hardly detectable in the peripheral blood of healthy individuals, procoagulant TF-bearing microparticles circulating at elevated levels are often associated with thrombotic propensity [11, 12]. In contrast with the well-documented thrombogenicity of microparticle-associated $\mathrm{TF}$, the physiological activity of the soluble fluid-phase TF is quite unclear because TF requires association with anionic lipids to become procoagulant [13].

Prominent among the virulence factors involved in $P$. aeruginosa pathogenicity is ExoU, a toxin with phospholipase A2 (PLA2) activity that is injected directly into eukaryotic cells via the type III secretory system [14]. Previous studies from the current authors' group have highlighted the ability of ExoU to induce the release of large amounts of free arachidonic acid and eicosanoids, and to elicit a potent inflammatory response of airway cells $[15,16]$.

Studies carried out by KURAHASHI et al. [17] showed the critical role played by ExoU in the pathogenesis of sepsis occurring following $P$. aeruginosa pneumonia. However, the ability of ExoU to activate the coagulation cascade in animal airways has not yet been investigated.

The present study addressed the question of whether ExoU would upregulate the expression of TF by airway epithelial respiratory cells, thereby modulating their procoagulant activity. The current study was further motivated by studies reporting the modulation of TF-dependent procoagulant properties of human monocytes by arachidonic acid and both lipoxygenase- and cyclooxygenase-derived eicosanoids [18, 19].

\section{MATERIAL AND METHODS}

\section{Bacterial strains and culture conditions}

The laboratory $P$. aeruginosa PA103 strain and its ExoUdeficient PA103AexoU mutant [15] were used throughout the current study. An exoU-depleted mutant complemented with an exoU gene with a site-specific mutation in the PLA2 catalytic site (PA103 DUT/S142A), a generous donation of A. Hauser (Northwestern University, Chicago, IL, USA), was also used.

Bacteria were grown in Luria-Bertani broth at $37^{\circ} \mathrm{C}$ for $14-16 \mathrm{~h}$ under mild agitation, harvested by centrifugation and resuspended in M-199 cell culture medium (Sigma-Aldrich, St Louis, MO, USA) such that the suspension's absorbance at a wavelength of $640 \mathrm{~nm}$ was 0.1 , corresponding to $\sim 10^{8}$ colony forming units $(\mathrm{CFU}) \cdot \mathrm{mL}^{-1}$.

\section{Cell culture and infection}

Human bronchial epithelial cells from the BEAS-2B line were cultured in M-199 cell culture medium containing 10\% foetal calf serum, glutamine and antibiotics (complete culture medium). Confluent cultures were trypsinised, cells were suspended in complete culture medium, seeded in 24-well $\left(0.4 \times 10^{5}\right.$ cells $^{*}$ well $\left.^{-1}\right)$ tissue culture plates and cultured for $48 \mathrm{~h}$. Cells were then infected at a multiplicity of infection of $\sim 100$ bacteria $\cdot$ cell $^{-1}$. Since translocation of effector proteins from the type III secretory system depends on close contact between bacteria and host cells, bacteria were centrifuged $(1,000 \times g$ for $10 \mathrm{~min})$ onto the cell monolayers prior to incubation at $37^{\circ} \mathrm{C}$ for $1 \mathrm{~h}$. Cells were then immediately processed or incubated with culture medium containing gentamicin at $300 \mu \mathrm{g} \cdot \mathrm{mL}^{-1}$ for different periods. In some assays, bacteria were treated for $30 \mathrm{~min}$ with $100 \mu \mathrm{M}$ of the cytosolic PLA2 inhibitor methyl arachidonyl fluorophosphonate (MAFP), before addition to the cell cultures.

\section{Detection of TF mRNA by RT-PCR}

Total RNA was isolated from noninfected (control) or from BEAS-2B cells infected for $1 \mathrm{~h}$ using the QIAGEN RNeasy kit (QIAGEN, Hilden, Germany). cDNA was synthesised from total RNA by reverse transcription with the SuperScript ${ }^{\mathrm{TM}}$ First-Strand Synthesis System for RT-PCR (Invitrogen, Carlsbad, CA, USA) according to the manufacturer's instructions. cDNA was subjected to the following PCR conditions: denaturation at $95^{\circ} \mathrm{C}$ for $2 \mathrm{~min}$; and 30 (TF) or 25 ( $\beta$-actin) cycles of denaturation at $95^{\circ} \mathrm{C}$ for $45 \mathrm{~s}$, annealing at $50^{\circ} \mathrm{C}$ for $45 \mathrm{~s}$ and extension at $72^{\circ} \mathrm{C}$ for $45 \mathrm{~s}$. An additional extension step of $5 \mathrm{~min}$ at $72^{\circ} \mathrm{C}$ was carried out after the last cycle. The primers used in the reactions were: $5^{\prime}$-CCC GAA CAG TTA ACC GGA AGA-3' (TF sense); 5'-GCT CCA ATG ATG TAG AAT ATT TCT CTG A-3' (TF antisense); 5'-CCT CGC CTT TGC CGA TCC-3' ( $\beta$-actin sense) and 5'-GGA TCT TCA TGA GGT AGT CAG TC-3' ( $\beta$-actin antisense). PCR products were subjected to electrophoresis in a $2 \%$ agarose gel and densitometry was performed using LabImage software (Kaplan $\mathrm{GmbH}$, Halle, Germany).

\section{Detection of cell-associated TF}

The IMUBIND Tissue Factor ELISA kit (American Diagnostica, Stamford, CT, USA) was used to quantify TF in both extracts and supernatants from BEAS-2B cells infected for $1 \mathrm{~h}$ and incubated with the gentamicin-containing culture medium for an additional $2 \mathrm{~h}$ as well as in extracts from mice lungs at $24 \mathrm{~h}$ after intratracheal inoculation of $P$. aeruginosa suspensions, according to the manufacturer's instructions.

\section{Detection of membrane-bound TF}

Cell cultures infected for $1 \mathrm{~h}$ and incubated with the gentamicin-containing culture medium for additional $2 \mathrm{~h}$ were detached from the microplate wells with $0.05 \%$ EDTA, fixed with $4 \%$ paraformaldehyde in PBS, incubated with an anti-TFfluorescein isothiocyanate (FITC) complex (American Diagnostica) and analysed with a FACScalibur flow cytometer (Becton Dickinson, Franklin Lakes, NJ, USA).

\section{Functional relevance of cell-associated TF}

TF exists in microdomains on the cell surface with a mixed population of active and encrypted proteins. Whereas TF can 
be detected on the surface of unactivated cells, its full procoagulant activity is not. Therefore, two different approaches were used to investigate whether ExoU would induce airway cells to exhibit a procoagulant activity. In both, cells were infected for $1 \mathrm{~h}$ and incubated with the gentamicin-containing culture medium for an additional $2 \mathrm{~h}$. In the first approach, noninfected and infected cells were dissociated from the microplate wells with $0.05 \%$ EDTA, rinsed, suspended in PBS and $10^{3}$ cells were incubated with pooled citrated plasma obtained from human volunteers at $37^{\circ} \mathrm{C}$ for $2 \mathrm{~min}$. Clot time was measured in duplicate as time necessary for clot to form (detected by the naked eye) following the addition of $25 \mathrm{mM} \mathrm{CaCl}_{2}$.

In the second approach, control and infected cells were lysed by repeated freeze-thaws in a buffer containing $50 \mathrm{mM}$ Tris$\mathrm{HCl}, 100 \mathrm{mM} \mathrm{NaCl}, 0.1 \%$ Triton X-100, pH 7.4, and TF was extracted in the buffer for $18 \mathrm{~h}$ at $4^{\circ} \mathrm{C}$ under mild agitation. Procoagulant activity in cell lysates was then assessed with the Actichrome TF activity kit (American Diagnostica), according to the manufacturer's instructions.

\section{Detection of TF-containing microparticles}

Supernatants from controls and from cultures infected for $1 \mathrm{~h}$ and treated with the gentamicin culture medium for $2 \mathrm{~h}$ were centrifuged to remove cell debris. Supernatants were further centrifuged at $17,500 \times g$ for $30 \mathrm{~min}$ at $15^{\circ} \mathrm{C}$, to obtain microparticles. Pellets were washed, treated simultaneouly with anti-TF-FITC and annexin V-Alexa Fluor 647 complexes (Invitrogen) for $30 \mathrm{~min}$ in ice and washed once. Microparticles were resuspended in PBS containing $1 \%$ bovine serum albumin and analysed for $1 \mathrm{~min}$ in a FACScalibur flow cytometer. The region corresponding to shed microparticles was gated in side scatter versus fluorescent intensity dot plot representations by using, as reference, a mix of fluorescent beads of diameters to cover the microparticles $(0.5$ and $0.9 \mu \mathrm{m})$ and platelets (3.0 $\mu \mathrm{m}$; Megamix; Biocytex, Marseille, France), according to the manufacturer's instructions.

\section{Detection of supernatant procoagulant activity}

Procoagulant activity of cell culture supernatants obtained after centrifugation for $10 \mathrm{~min}$ to remove cell debris (microparticle-enriched supernatants) or after further centrifugation at $17,500 \times g$ for $30 \mathrm{~min}$ (microparticle-free supernatants) was assessed with a chromogenic assay (Actichrome TF activity kit).

\section{In vivo assays}

Female Swiss mice aged 8-12 weeks were anaesthetised with a mixture of ketamine $\left(65 \mathrm{mg} \cdot \mathrm{kg}^{-1}\right)$ and xylazine $\left(13 \mathrm{mg} \cdot \mathrm{kg}^{-1}\right)$ administered i.p., and $10^{4}$ CFU of PA103 or PA103 4 exoU in $50 \mu \mathrm{L}$ LPS-free saline were instilled into their tracheas. At $24 \mathrm{~h}$ post-infection, mice were euthanised by i.p. injection of sodium pentobarbital. Their airways were then washed once with $1 \mathrm{~mL}$ of PBS. Lungs were next washed free of blood by perfusing the heart and lungs with $10 \mathrm{~mL}$ of cold PBS and kept at $-70^{\circ} \mathrm{C}$ for ulterior treatment with lysis buffer, ELISA detection of TF and assessment of procoagulant activity, using the Actichrome TF activity kit, according to the manufacturer's instructions. All animal experiments were approved by the Animal Ethics Committee of the State University of Rio de Janeiro (Rio de Janeiro, Brazil).

\section{Statistical analysis}

Statistical analysis was performed using a one-way ANOVA with Bonferroni's test to determine significant statistical differences between groups, unless otherwise stated. p-values $<0.05$ were deemed to be significant.

\section{RESULTS}

\section{ExoU enhanced the expression of TF by $\mathrm{P}$. aeruginosa- infected cells}

RT-PCR was performed to investigate whether P. aeruginosa infection and/or ExoU production could alter TF mRNA levels in airway epithelial cells. The data shown in fig. 1 show that TF transcripts were constitutively present in BEAS-2B cells and that infection for $1 \mathrm{~h}$ with the ExoU-producing wild-type bacteria increased the level of mRNA by $\sim 45 \%$, in comparison with noninfected and PA103AexoU-infected cells.

Infection with PA103 for $3 \mathrm{~h}$ increased the baseline expression of TF protein approximately 1.5-fold, as detected by ELISA assays in control culture lysates. Previous bacterial treatment with the PLA2 inhibitor MAFP reduced significantly the expression of TF by PA103-infected cells. Consistent with the
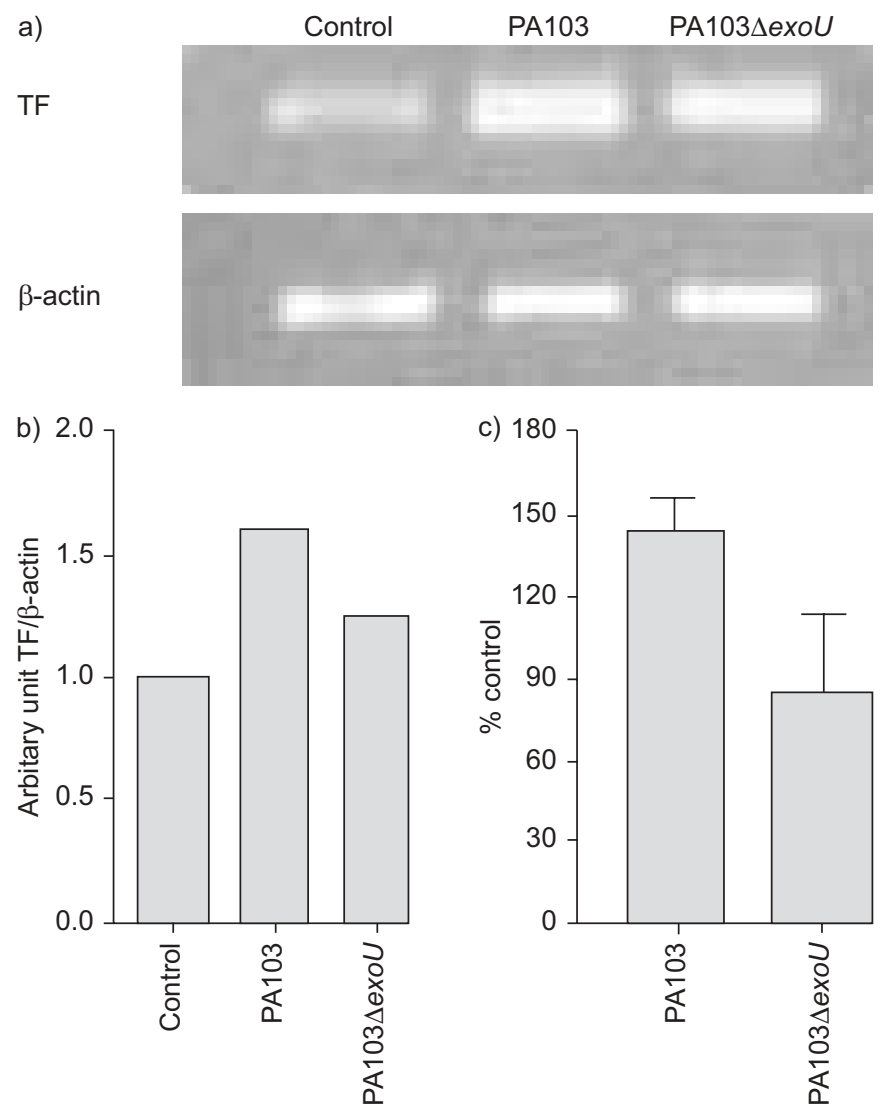

FIGURE 1. a) RT-PCR analysis of tissue factor (TF) and $\beta$-actin mRNA transcripts in control noninfected cells and in cells infected with the ExoU-producing PA103 bacterial strain and the ExoU-deficient mutant PA103AexoU, representative of four analyses carried out with similar results. b) Ratio of TF to $\beta$-actin interna control density in the agarose gel shown in (a). c) Increase of the relative TF/ $\beta$-actin transcript densities in PA103 and PA103 LexoU-infected cells in comparison with the relationship obtained in control cells. Data are presented as mean \pm SD of four different assays. 
role of ExoU PLA2 activity in the modulation of TF expression, no increase in TF was detected in cells infected with bacteria deficient in exoU or with mutated PLA2 catalytic activity site (fig. 2a). The enhanced expression of TF in PA103-infected cells was further confirmed by fluorescence-activated cell sorting analysis of cells labelled with a monoclonal anti-TF antibody (fig. 2b).

The biological relevance of enhanced TF expression by PA103infected cells was investigated next. As shown in figure 3a, clotting time was significantly lower and procoagulant activity was significantly higher in cells infected with ExoU-producing bacteria than in control cells or in cells infected with the exoU bacterial mutant (fig. $3 a$ and b).
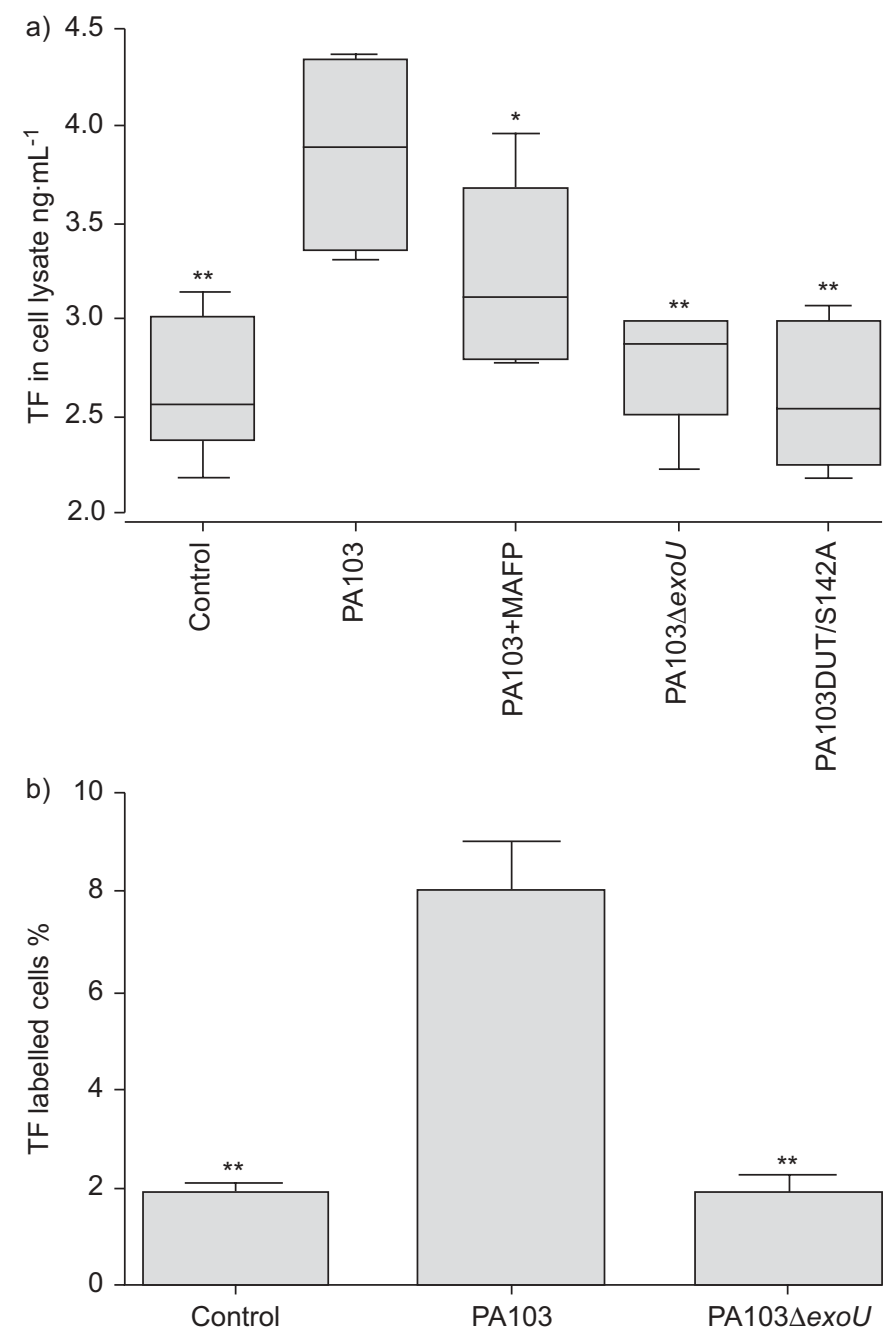

FIGURE 2. a) Concentration of tissue factor (TF) in lysates from control and Pseudomonas aeruginosa-infected airway epithelial cells. The boxes extend from the 25th-75th percentiles, with the horizontal line at the median value. Whiskers show the range of the data. b) Percentage of cells expressing surface TF, as determined by fluorescence-activated cell sorting analysis. Data are presented as mean $\pm S D$ of the results from two different assays carried out in triplicate. *: $p<0.05 ; * *: p<0.01$ when data were compared with the results obtained from PA103-infected cells.

\section{ExoU also enhanced the release of TF-bearing microparticles from infected cells}

The potential of ExoU to modulate the release of TF from cell plasma membranes was assessed by ELISA. Figure 4a shows that the concentration of TF in supernatants from PA103infected cells was about five-fold higher than in supernatants from control and PA103AexoU-infected cultures. Infection with the ExoU-producing bacteria also enhanced the release of microparticles from cell membranes (fig. 4b), and the percentage of microparticles expressing TF at their surfaces was significantly higher in supernatants from PA103-infected cells (fig. 4c). More importantly, a higher percentage of microparticles released after PA103 infection, besides exhibiting surface $\mathrm{TF}$, was shown to bind annexin $\mathrm{V}$ (fig. $4 \mathrm{~d}$ ), a protein known for its interaction with negatively charged phospholipids, such as phosphatidylserine, one of the essential lipid cofactors for clotting. These TF-positive/annexin V-positive microparticles are likely to provide a catalytic surface promoting the assembly of the enzyme complexes of the coagulation cascade.

\section{TF-bearing microparticles, but not soluble TF, accounted for the procoagulant activity of cell culture supernatants}

Since conflicting results have been published relating to the contribution of the soluble isoform $[9,10,14]$ and full-length TF
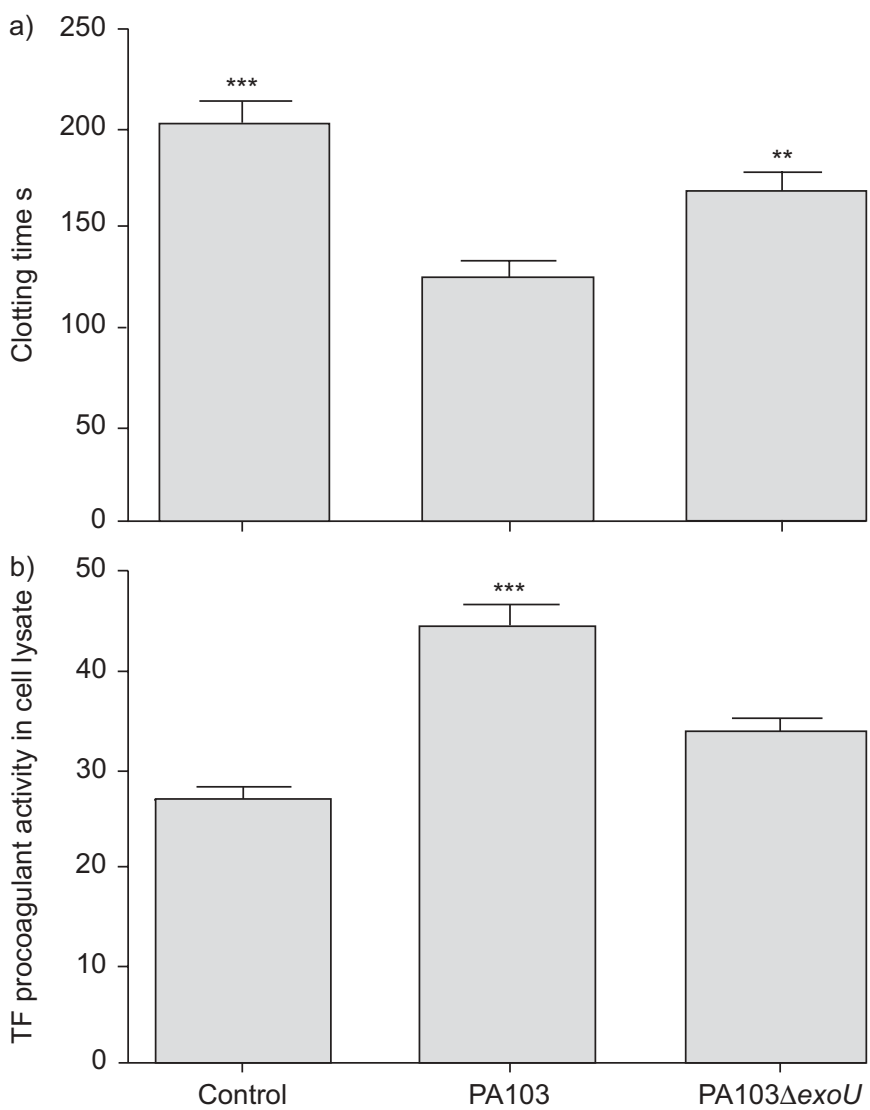

FIGURE 3. Clotting time (a) and procoagulant activity (b) of control and Pseudomonas aeruginosa-infected airway epithelial cells. Data are presented as mean $\pm S D$ of the results from three different assays carried out in tripicate **: $p<0.01$; ***: $p<0.001$ when data were compared with the results obtained from PA103-infected cells 

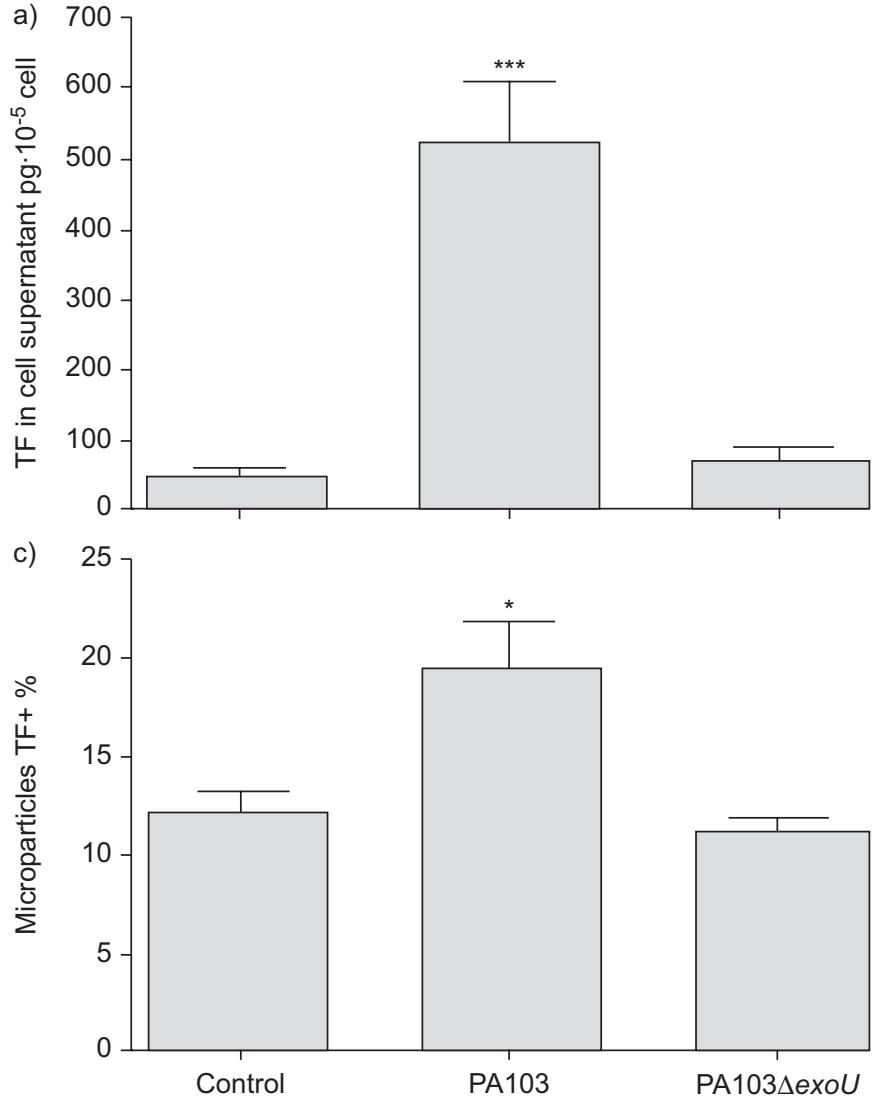
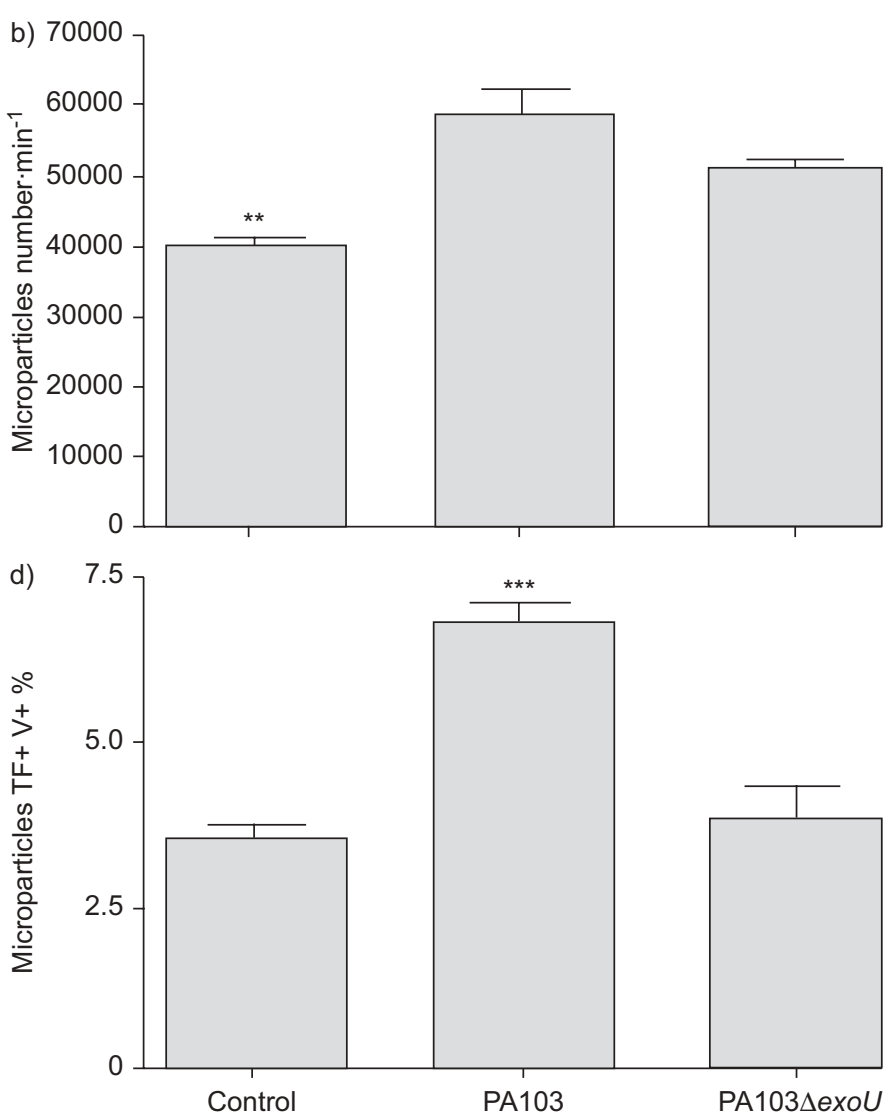

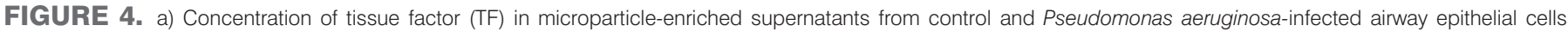

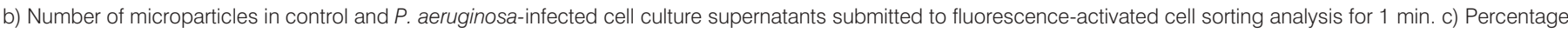

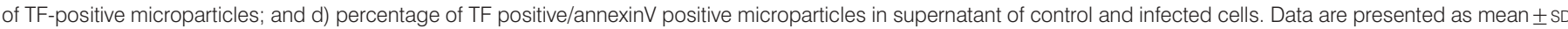

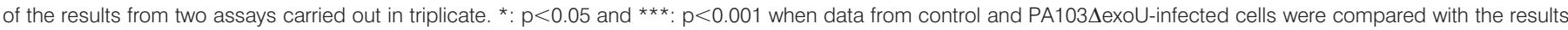
from PA103-infected cultures ( $a, c, d) .{ }^{*}: p<0.01$ when the results from control cultures were compared with those from cells infected with the wild-type bacteria (b).

associated with microparticles to thrombogenicity of extracellular fluids, the impact of each one on the procoagulability of airway epithelial cell supernatants was investigated. Figure 5 shows the results obtained with a chromogenic assay and shows that infection with the ExoU-producing bacteria led to an $\sim 1.8$-fold rise in TF activity in microparticle-enriched supernatants from PA103-infected cells, compared with noninfected cultures. When the effect of ExoU was assessed in microparticle-free culture supernatants, the thrombogenicity in supernatant from PA103-infected cultures dropped from $35.9 \pm 3.1 \mathrm{pM}$ to $0.4 \pm 0.2 \mathrm{pM}$. No activity was detected in supernatants from controls and from cells infected with the ExoU-deficient mutant (data not shown). These results clearly show that TF-bearing microparticles, but not soluble TF, accounted for the thrombogenicity of cell culture supernatants.

\section{Lung samples from mice infected intratracheally with ExoU-producing bacteria exhibited higher TF concentration and thrombogenicity}

To ascertain the biological relevance of the in vitro results, the in vivo effect of ExoU on TF expression by lung cells was investigated. As shown in figure 6a, TF concentration in lung parenchyma from mice infected with the ExoU-producing PA103 strain was approximately three-fold higher than in lungs from mice infected with the ExoU-deficient bacteria. Lysates of PA103-infected lung parenchyma also exhibited a higher procoagulant activity (fig. 6b), consistent with data obtained in in vitro assays.

\section{DISCUSSION}

The current study demonstrated the ability of ExoU to induce thrombogenic properties in airway epithelial cells through active transcriptional modulation of TF expression, and to enhance the release of procoagulant microparticles from infected cells. The present authors are unaware of any prior investigation that directly addressed this possibility. Since in vitro results were supported by similar finding with lysates of the lung parenchyma of PA103-infected mice, the current authors speculate that this ability may contribute to the clinical significance of this toxin, considered to be a marker for highly virulent $P$. aeruginosa isolates recovered from patients with hospital-acquired pneumonia [20] and bacteraemia [21].

Consistent with other studies, BEAS-2B airway cells were shown to express TF mRNA and protein constitutively. Such TF expression is likely to provide airway epithelial cells with the capability to generate a provisional fibrin matrix to favour their migration during the wound-healing process following bronchial epithelium injury, independently of plasma proteins [22]. 


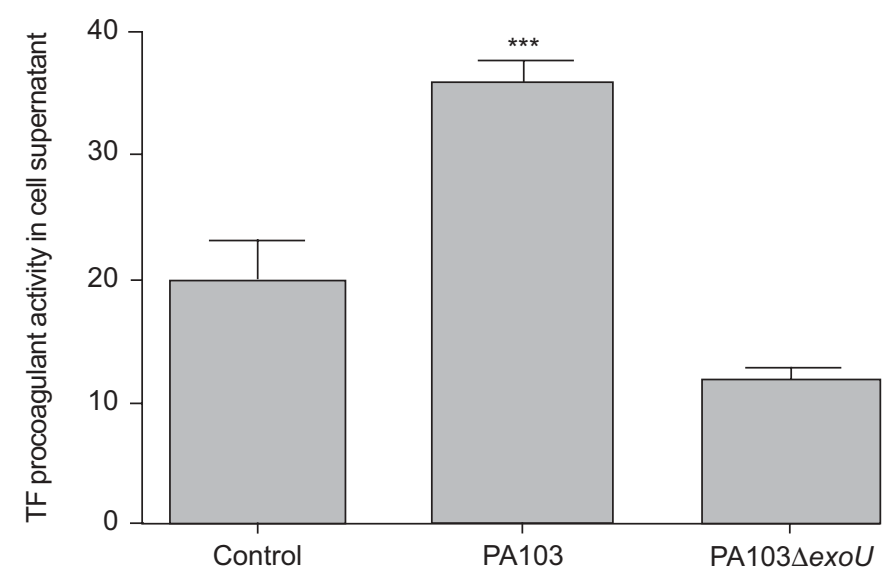

FIGURE 5. Procoagulant activity of microparticle-enriched supernatant of control and Pseudomonas aeruginosa-infected airway epithelial cells. Data are presented as mean $\pm S D$ of the results from two assays carried out in triplicate. $* * *: p<0.001$ when data were compared with the results from PA103-infected cultures.

Therefore, the initiation of clot formation by TF-bearing airway cells is believed to be essential for a rapid initial repair process. Ulterior leakage of plasma protein (including fibrinogen) into the airways would favour a potentially harmful propagation of the coagulation. Transitional fibrin itself may lead to deleterious effects by providing a matrix on which fibroblasts can also migrate and produce collagen, leading to accelerated pulmonary fibrosis. Additionally, fibrin can enhance local inflammatory response by increasing vascular permeability and activating endothelial cells to produce proinflammatory cytokines and other mediators. Thus, the local fibrin formation by TF-bearing airway cells may become a pathological event. Indeed, fibrin deposition in the lung parenchyma and airspaces is considered to be a hallmark of acute lung injury and acute respiratory distress syndrome [23].

The bronchoalveolar procoagulant response during severe infection has been shown to depend also on dysfunctional local anticoagulant pathways (e.g. antithrombin and the protein $C$ system) and fibrinolytic activity, caused by high levels of plasminogen activator inhibitor-1 and reduced levels of plasminogen activator activity, as described by $\mathrm{CHOI}$ and $\mathrm{CO}^{-}$ workers $[24,25]$ in patients with ventilator-asociated pneumonia and experimental pneumonia in rats.

In the current report, cells infected with the ExoU-producing $P$. aeruginosa strain exhibited significantly higher TF content. Previous treatment of bacteria with MAFP, a PLA2 inhibitor, reduced their ability to upregulate TF production significantly, consistent with the phospholipase activity of ExoU. These in vitro findings were validated by the demonstration that both TF concentration and procoagulant activity in the lung parenchyma of PA103-infected mice were substantially higher than in the parenchyma of mice infected with the exoU mutant. Based on these results, it can be speculated that overexpression of TF elicited by bacterial infection can initiate local coagulation, contributing at least partially to the severe lung injury and increased mortality described in both patients [20] and experimental animals [17] with pneumonia caused by ExoUproducing $P$. aeruginosa strains.
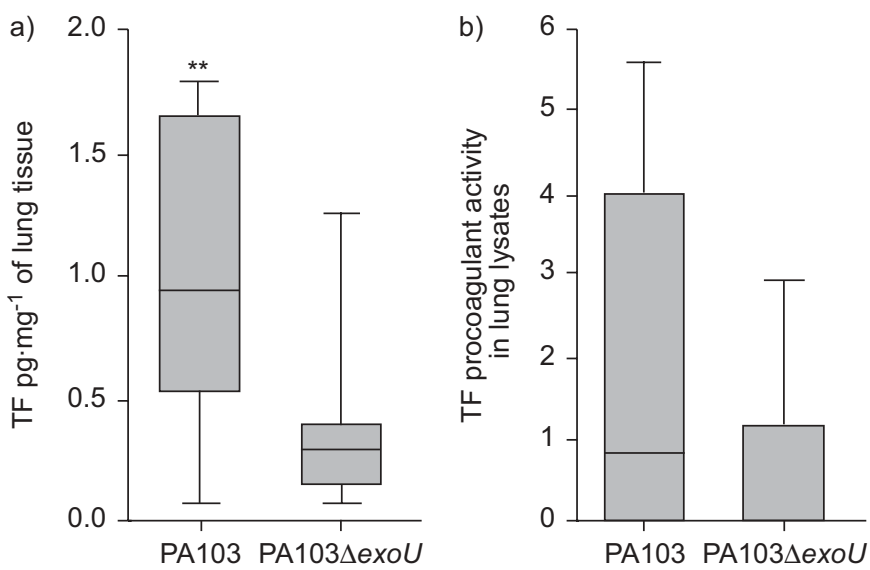

FIGURE 6. a) Concentration of tissue factor (TF) and b) procoagulant activity detected in lung lysates from control and Pseudomonas aeruginosa-infected mice. The boxes extend from the 25th-75th percentiles, with the horizontal line at the median value. Whiskers show the range of the data obtained from animals infected with PA103 $(n=10)$ or PA103 $\operatorname{exoU}(n=13)$. **: $p<0.01$ when data were compared using the Mann-Whitney test.

Besides being expressed at plasma membranes, TF can also circulate associated with microparticles released from different cell types upon activation and/or damage. There is increasing evidence that microparticles carry proinflammatory cell membrane lipids and glycoproteins testifying to their cellular origin that can be transferred to neighbouring or remote cells bearing appropriate counter-receptors for ligands they harbour. The cellular effects depend on microparticle membrane and cytoplasmic composition and the nature of target cells, and can be as different as cell activation, enhancement of monocyte-endothelium adhesiveness, vascular dysfunction, induction of apoptosis and others [11, 12]. The implication of microparticles in inflammation is also well documented. For instance, microparticles are a source of substrates of secretory PLA2 for generation of lysophosphatidic acid, a potent proinflammatory mediator and platelet agonist. Interestingly, in sepsis, elevated levels of PLA2 activity in bronchoalveolar lavage fluids have been shown to have a prognostic value [26].

In the present study, ExoU was also shown to augment both the number and the proportion of TF- and posphatidylserinebearing microparticles released by airway epithelial cells, and in vitro assays showed that these TF-bearing microparticles were procoagulant. It is conceivable that a similar in vivo augmentation of microparticle release would represent a second mechanism by which ExoU would increase the thrombotic propensity of $P$. aeruginosa-infected airway epithelial cells. However, knowing that microparticles are endowed with the potential to disseminate a wide range of different biological information, the pathophysiological role played by ExoU-induced microparticles may not be restricted to their procoagulant potential. For instance, microparticles have been shown both to be a rapid substrate for peroxidation and to carry biologically active oxidised phospholipids that elicit specific responses in endothelial cells [27]. Excitingly, the present authors have previously demonstrated the ability of ExoU to induce a significant peroxidation of cell membrane lipids from PA103-infected endothelial cells [28]. Increased 
release of oxidised microparticles from $P$. aeruginosa-infected cells is likely to contribute to the progression of inflammatory processes elicited in the course of $P$. aeruginosa infection.

Different human pathogens [29, 30] have been shown to modulate the expression of tissue factor by monocytes and endothelial cells, thereby promoting vascular thrombosis but, to the current authors' knowledge, this is the first study reporting the bacterial up-regulation of tissue factor in airway epithelial cells. However, it is conceivable that ExoU may similarly enhance tissue factor expression by endothelial cells and that this ExoU ability during Pseudomonas aeruginosa bacteraemia could serve as a virulence factor promoting a hypercoagulable state frequently detected in sepsis. Studies to examine this hypothesis are currently in progress.

\section{ACKNOWLEDGEMENTS}

The current authors wish to thank M.A. Pereira da Silva, M. Jones and C. Barbosa for their technical assistance.

\section{REFERENCES}

1 Levi M, Keller TT, van Gorp E, Cate H. Infection and inflammation and the coagulation system. Cardiovasc Res 2003; 60: 26-39.

2 Martin GS, Bernard GR. Airway and lung in sepsis. Intensive Care Med 2001; 27: Suppl. 1, S63-S79.

3 Seeger W, Hübel J, Klapettek K, et al. Procoagulant activity in bronchoalveolar lavage of severely traumatized patients - relation to the development of acute respiratory distress. Thromb Res 1991; 61: 53-64.

4 Chu AJ. Tissue factor mediates inflammation. Arch Biochem Biophys 2005; 15: 123-132.

5 Welty-Wolf KE, Carraway MS, Ortel TL, Piantadosi CA. Coagulation and inflammation in acute lung injury. Thromb Haemost 2002; 88: 17-25.

6 Østerud B, Bjørklid E. Sources of tissue factor. Semin Thromb Hemost 2006; 32: 11-23.

7 Mackman N, Sawdey MS, Keeton MR, Loskutoff DJ. Murine tissue factor gene expression in vivo: tissue and cell specificity and regulation by LPS. Am J Pathol 1993; 143: 76-84.

8 Bastarache JA, Wang L, Geiser T, et al. The alveolar epithelium can initiate the extrinsic coagulation cascade through expression of tissue factor. Thorax 2007; 62: 608-616.

9 Bogdanov Y. Alternatively spliced human tissue factor: a circulating soluble thrombogenic protein. Nat Med 2003; 9: 458-462.

10 Giesen PL, Rauch U, Bohrmann B, et al. Blood-borne tissue factor: another view of thrombosis. Proc Natl Acad Sci USA 1999; 96: 2311-2315.

11 Morel O, Toti F, Hugel B, Freyssinet JM. Cellular microparticles: a disseminated storage pool of bioactive vascular effectors. Curr Opin Hematol 2004; 11: 156-164.

12 Hugel B, Martinez MC, Kunzelmann C, Freyssinet JM. Membrane microparticles: two sides of the coin. Physiol 2005; 20: 22-27.

13 Sturk-Maquelin KG, Niewland R, Romijn FP, Eijsman L, Hack CE, Sturk A. Pro- and non-coagulant forms of non-cell bound tissue factor in vivo. J Thromb Haemost 2003; 1: 1920-1926.
14 Sato H, Frank DW, Hillard CJ, et al. The mechanism of action of the Pseudomonas aeruginosa-encoded type III cytotoxin, ExoU. EMBO J 2003; 22: 2959-2969.

15 Saliba AM, Nascimento DO, Silva MC, et al. Eicosanoidmediated proinflammatory activity of Pseudomonas aeruginosa ExoU. Cell Microbiol 2005; 7: 1811-1822.

16 Plotkowski MC, Brandão BA, de Assis MC, et al. Lipid body mobilization in the ExoU-induced release of inflammatory mediators by airway epithelial cells. Microb Pathog 2008; 45: 30-37.

17 Kurahashi K, Kajikawa O, Sawa T, et al. Pathogenesis of septic shock in Pseudomonas aeruginosa pneumonia. J Clin Invest 1999; 104: 743-750.

18 Eilertsen KE, Olsen JO, Osterud B. Ex-vivo regulation of endotoxin-induced tissue factor in whole blood by eicosanoids. Blood Coagul Fibrinolysis 2003; 14: 41-48.

19 Cadroy Y, Dupouy D, Boneu B. Arachidonic acid enhances the tissue factor expression of mononuclear cells by the cyclooxygenase 1 pathway. J Immunol 1998; 160: 6145-6150.

20 Schulert GS, Feltman H, Rabin SDP, et al. Secretion of the toxin ExoU is a marker for highly virulent Pseudomonas aeruginosa isolates obtained from patients with hospitalacquired pneumonia. J Infect Dis 2003; 188: 1695-1706.

21 Berthelot $\mathrm{P}$, Attree I, Plesiat $\mathrm{P}$, et al. Genotypic and phenotypic analysis of the type III secretion system in a cohort of Pseudomonas aeruginosa bacteremia isolates: evidence for a possible association between $\mathrm{O}$ serotypes and exo genes. J Infect Dis 2003; 188: 512-518.

22 Perrio MJ, Ewen D, Trevethick MA, Salmon GP, Shute JK. Fibrin formation by wounded bronchial epithelial ccell layers in vitro is essential for normal epithelial repair and independent of plasma proteins. Clin Exp Allergy 2007; 37: 1688-1700.

23 Wygrecka M, Jablonska E, Guenther A, Preissner KT, Markart P. Current view on alveolar coagulation and fibrinolysis in acute inflammatory and chronic interstitial lung diseases. Thromb Haemost 2008; 99: 494-501.

24 Choi G, Vlaar APJ, Schouten M, et al. Natural anticoagulants limit lipopolysaccharide-induced pulmonary coagulation but not inflammation. Eur Respir J 2007; 30: 423-428.

25 Choi G, Hofstra JJH, Roelofs JJTH, et al. Recombinant human activated protein $\mathrm{C}$ inhibits local and systemic activation of coagulation without influencing inflammation during Pseudomonas aeruginosa pneumonia in rats. Crit Care Med 2007; 35: 1362-1368.

26 Arbibr L, Vial D, Touqui L. Phospholipase A2 and acute respiratory distress syndrome. In: Uhl W, Nevalainem TJ, Büchler MW, eds. Phospholipase A2: basic and clinical aspects in inflammatory diseases. Prog Surg vol. 24. Basel, Karger, 1997; pp. 79-87.

27 Huber J, Vales A, Mitulovic G, et al. Oxidized membrane vesicles and blebs from apoptotic cells contain biologically active oxidized phospholipids that induce monocyteendothelial interactions. Arterioscler Thromb Vasc Biol 2002; 22: 101-107.

28 Saliba AM, de Assis MC, Nishi R, et al. Implications of oxidative stress in the cytotoxicity of Pseudomonas aeruginosa ExoU. Microbes Infect 2006; 8: 450-499.

29 Bryant AE, Hayes-Schroer SM, Stevens DL. M type 1 and 3 group A streptococci stimulate tissue factor-mediated 
procoagulant activity in human monocytes and endothelial cells. Infect Immun 2003; 71: 1903-1910.

30 Mattsson E, Heying R, van de Gevel JS, Hartung T, Beekhuizen H. Staphylococcal peptidoglycan initiates an inflammatory response and procoagulant activity in human vascular endothelial cells: a comparison with highly purified lipoteichoic acid and TSST-1. FEMS Immunol Med Microbiol 2008; 52: 110-117. 